\title{
Cerebral vasculitis - a rare presentation of antineutrophil cytoplasmic antibody associated vasculitis relapse
}

\author{
Sara Daniela Rodrigues, Ana Maria Ventura, Daniela Lopes, Susana Pereira, Maria Clara Almeida, Ana Marta Gomes, João Carlos Fernandes \\ Nephrology Department, Centro Vila Nova de Gaia e Espinho Hospital, Vila Nova de Gaia, Portugal.
}

\section{ABSTRACT}

Antineutrophil cytoplasmic antibody (ANCA) associated vasculitis commonly involves kidney, lung, upper respiratory tract, skin, gastrointestinal and occasionally peripheral nervous system. Central nervous system is less commonly affected and is generally part of a multi-organ scenario. We present a case of a 21-year-old female with chronic kidney disease secondary to myeloperoxidase ANCA associated vasculitis, diagnosed at age 19. She then presented with pulmonary-renal syndrome with stage 5 CKD. Three months' cyclophosphamide and prednisone were instituted, followed by azathioprine, but she remained dialysis dependent. After 2 years, while on maintenance treatment, she was admitted to the emergency department with tonic-clonic seizures. She had started oral ciprofloxacin 2 days before for acute gastroenteritis. No illicit drugs were noticed. Blood tests showed an increased myoglobin but were otherwise unremarkable. No anomalies were reported on cerebral computed tomography or cerebrospinal fluid analysis. Magnetic resonance imaging showed diffuse cortico-subcortical lesions with $\mathrm{T} 2$ and proton density hypersignal suggesting recent ischemia, and segmental irregularities of various cerebral and vertebral arteries, consistent with small and medium size vessel vasculitis. Intravenous methylprednisolone and oral cyclophosphamide were started. Anti-myeloperoxidase ANCA levels were increased (>200 RU/mL), reinforcing our diagnostic hypothesis of central nervous system (CNS) recurrence of ANCA vasculitis. The patient had a favorable clinical course, with no neurological sequelae.

Conclusions: This is a case of ANCA associated vasculitis that presented with kidney and lung but no CNS involvement and recurred with primarily cerebral disease. Favorable outcome was seen with standard immunosuppression. Awareness of this rare but severe complication is critical for timely recognition and prompt treatment.

Key words: ANCA, antineutrophil cytoplasmic antibody, Central Nervous System Vasculitis, relapse, vasculitis.

\section{INTRODUCTION}

Antineutrophil cytoplasmic antibody (ANCA) associated vasculitis (AAV) is a collection of relatively rare autoimmune diseases of unknown cause, characterized by inflammatory cell infiltration causing necrosis of small vessels, with few or no immune deposits ${ }^{1-3}$. Serologically, it is associated with a specific ANCA for myeloperoxidase (MPO-ANCA) or proteinase 3 (PR3-ANCA). Clinical presentation comprises a wide spectrum of manifestations from the common nephrological, respiratory, dermatological, gastrointestinal symptoms to infrequent neurological and cardiac complications $s^{1-3}$. The serotype should be accompanied by the clinicopathologic variant if this can be determined: microscopic polyangiitis, granulomatosis with polyangiitis (Wegener), eosinophilic granulomatosis with polyangiitis (Churg-Strauss), or renallimited vasculitis. AAV have a peak incidence at $65-75$ years old, but may occur at any age, with a slight male predominance ${ }^{2}$. Approximately one fourth to one half of patients with AAV will experience a relapse within several years ${ }^{2}$. Recurrence rate has been reported as $0.16-0.20$ / patient/year in non-end stage renal disease (ESRD) patients, and lower values in ESRD - 0.08/patient/year ${ }^{4}$. Relapses are diagnosed on the basis of clinical and pathologic evidence of recurrent disease. Some factors have been associated with higher recurrence risk, as PR3-ANCA (versus MPO), pulmonary or superior respiratory tract involvement, previous relapses and elevated ANCA titres, mainly when renal involvement is present ${ }^{5,6}$.
CNS involvement is an uncommon but serious complication and it often arises when vasculitis is active elsewhere ${ }^{7,8}$. It is thought to be caused by 3 distinct pathogenic mechanisms. First, granulomatous tissue may spread from the nasal or paranasal cavities and contiguously invade the adjacent structures, for example, orbit, optic nerve, meninges or pituitary gland. The second refers to remote granulomatous intracerebral lesions of the brain, meninges, cranial nerves, or parietal bone. Third, vasculitis may affect the cerebral or spinal cord vessels ${ }^{7}$. Clinical presentation may be characterized by headache, focal neurologic deficits, seizures, ischemic stroke or subarachnoid hemorrhage ${ }^{8}$. Diagnosis is usually based on clinical CNS manifestations and multiple ischemic (sometimes hemorrhagic) MR lesions mainly affecting the white matter. Treatment with glucocorticoids and cyclophosphamide usually improves clinical features and MRI lesions $2,3,8-10$.

\section{CASE REPORT}

We present a case of a 21-year-old female with chronic kidney disease (CKD) secondary to MPO-ANCA associated vasculitis. Past medical history was relevant for cigarette smoking (3 cigarettes/day). Vasculitis had been diagnosed at age 19. She then presented with severe kidney dysfunction, requiring dialysis, and mild bloody sputum, although no massive hemoptysis or severe respiratory impairment 
were present. ANCA titers were elevated (1/640), with MPO specificity (MPO-ANCA $>200.0 \mathrm{RU} / \mathrm{mL}$ ) and anti-glomerular basement membrane (GBM) autoantibodies were negative. Chest computed tomography (CT) showed patchy ground-glass opacities, without pleural effusion. Pauci-immune crescentic glomerulonephritis, with $90 \%$ of the glomeruli being sclerosed and $50 \%$ of interstitial fibrosis, was seen in the kidney biopsy (end stage kidney). Initial immunosuppressive therapy with pulse methylprednisolone $1 \mathrm{~g} /$ day for 3 days and oral cyclophosphamide $2 \mathrm{mg} / \mathrm{kg}$ per day was started. Considering the severe renal dysfunction and the pulmonary involvement, plasma exchange was also started, with 6 sessions performed in total. Her hemoglobin stabilized and she presented no more evidence of pulmonary hemorrhage. However, she remained dialysis dependent. After 3 months, a switch to maintenance immunosuppression with azathioprine was made. After 17 months, a surveillance chest CT showed comparatively subtle lung opacities in peribroncovascular areas. At this point, she had no recurrence of symptoms, although MPO-ANCA remained elevated $(185 \mathrm{RU} / \mathrm{mL})$. No further intercurrences have been observed since then.

After 2 years of AAV diagnosis, while on maintenance treatment with azathioprine $100 \mathrm{mg} /$ day, she was admitted to the emergency department with seizures. She had started oral ciprofloxacin 2 days before for acute gastroenteritis and had worsening hypertension for the previous 2 months. No illicit drugs were noticed. She presented in post ictal status and had two new ictal episodes during initial evaluation, described as focal motor evolving to bilateral tonic-clonic seizures. The remaining physical examination showed hypertension $(160 / 105 \mathrm{mmHg})$, heart rate of 101 beats per minute, peripheral oxygen saturation of $96 \%$, temperature $36.8^{\circ} \mathrm{C}$, with no abnormalities on cardiac, pulmonary, abdominal or skin examination. After anticonvulsant administration and once the ictal phase was resolved, no asymmetries in strength or sensitivity were detected. Blood tests showed leucocytosis with neutrophilia and raised myoglobin but were otherwise unremarkable. No drug was detected in the urine test except for benzodiazepines (diazepam was used for seizure treatment on admission). Cerebral computed tomography was normal. Cerebrospinal fluid (CSF) analysis showed only erythrocytes, with probable traumatic origin, but was otherwise unremarkable. Magnetic resonance imaging (MRI) showed diffuse cortico-subcortical lesions with $\mathrm{T} 2$ and proton density hypersignal, suggesting recent cortical ischemia, as well as segmental irregularities of medium and anterior cerebral branches, consistent with small and medium size vessel vasculitis (Figure 1). Chest CT scan was repeated, and subtle ground-glass opacities were, again, observed, suggesting possible lung bleeding, favouring the vasculitis flare scenario, but could also be attributed to cigarette associated bronchiolitis. In addition, centrilobular emphysema was also reported. MPO-ANCA remained elevated (>200 RU/mL).

Given the suspicion of CNS vasculitis, intravenous methylprednisolone was started, $1 \mathrm{~g} /$ day for 3 days, followed by oral prednisone, and oral cyclophosphamide $2 \mathrm{mg} / \mathrm{kg} /$ day.

The patient completed 6 months of oral cyclophosphamide, followed by azathioprine. A favourable clinical course was observed, with no neurological sequelae, and with normalization of MPO-ANCA. No other vasculitis relapses occurred since then.

\section{Figure 1}

Magnétic ressonance imaging (T2, proton density and angiopathy)
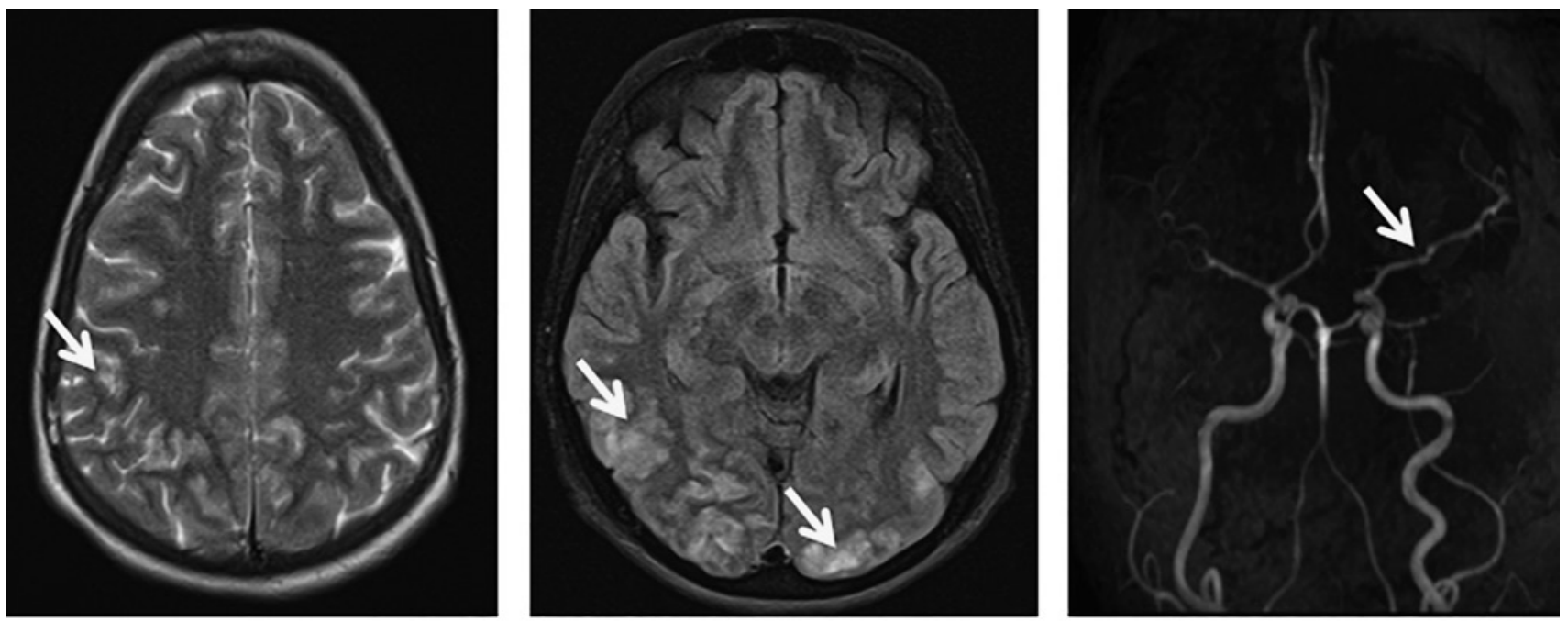

T2 (left) and proton density (middle) show diffuse hypersignal cortico-subcortical lesions located on the right posterior temporal lobe and on the frontal, parietal and occipital lobes, suggesting recent cortical ischaemia. Angiography (right) shows decreased diameter of medium and anterior cerebral arteries and vertebral artery, with segmental irregularities of $\mathrm{M} 1$ and $\mathrm{M} 2$ segments of medium cerebral arteries, consistent with small and medium size vessel vasculitis. 


\section{DISCUSSION}

We describe here a case of MPO-ANCA vasculitis that presented with end stage renal disease and pulmonary involvement, but no CNS symptoms, and recurred 2 years after diagnosis with primarily neurologic disease. CNS vasculitis is not very common and when it happens, it has been mostly described as part of a multisystemic phenotype ${ }^{2,3,9-11}$. In addition, CNS AAV recurrences, in general, have been reported after a first presentation with cerebral involvement as well 8,11 , although we found one case, described by Seror et al, of AAV associated pachymeningitis presenting with headache 6 years after the diagnosis of AAV that presented without SNC symptoms ${ }^{10}$. It is important to recognise that CNS manifestations in ANCA vasculitis may be due to vasculitis affecting the brain, but also to granulomata, hypertension, sepsis or coagulation disorders ${ }^{7-9}$. Distinguishing CNS vasculitis from other causes of cerebral lesions is challenging due to the scarcity of published data and difficulty in confirming this entity.

Here, the diagnosis of cerebral vasculitis was made on the basis of the clinical manifestations, characterised by seizures, by the abnormal brain MRI findings consistent with vasculitis 8,12 , and by the absence of other obvious causes of cerebral lesions. Infectious cause was ruled out on the basis of absence of fever or relevant increase inflammatory markers, as well as innocent CSF analysis. CSF may actually show pleocytosis, increased protein content, or both, in infections and in active CNS disease related to $\mathrm{AVV}^{10,13}$. However, CSF may also be entirely unremarkable ${ }^{14}$, as in our case.

Uncontrolled hypertension was present and, together with RMI findings, made us consider reversible posterior leukoencephalopathy syndrome (PRES) in our differential diagnosis. Cases of AAV associated PRES have been reported ${ }^{15-18}$. However, the asymmetrical nature of the lesions and the segmental irregularities of cerebral arteries branches with multiple stenosis was strongly evocative of vasculitis in our patient. This together with the clear response to the standard AAV immunosuppressant treatment reinforced our hypothesis of SNC relapse of AAV. CNS biopsy is considered the gold standard to diagnose intracranial vasculitis, but it is rarely performed because of its invasive nature and potential risks 19,20 .

Possible risk factors for recurrence in this patient could have included involvement of the lung at presentation and constantly positive ANCA titers, although there is still some controversy on the last issue ${ }^{21-23}$. Some authors believe that there is significant interindividual variability in the correlation between ANCA titer and activity of the disease ${ }^{21}$; others have found that ANCA is nearly always present in patients with renal involvement and correlates with activity, but is mainly negative in patients with nonrenal disease ${ }^{5}$. It has then been suggested that longitudinal ANCA measurements may be useful in patients with renal involvement but is less valuable in patients with nonrenal disease ${ }^{2}$. However, there is no established indication to guide treatment decisions only upon serial ANCA measurements during disease remission. Another precipitant for relapse could have been recent infection, possibly because acute-phase or cellular response to infection may enhance quiescent disease ${ }^{24,25}$. Our patient had been diagnosed with acute gastroenteritis two days prior to admission. She was treated with ciprofloxacin and we believe that the fluoroquinolone might have additionally contributed to the convulsive episodes, by lowering the convulsive threshold.

Finally, pulmonary changes observed at the last chest CT might suggest that some degree of pulmonary involvement could be present as well. The ground-glass opacities seen could represent lung bleeding. However, they could also represent bronchiolitis associated with cigarette smoking, as our patient remained an active smoker. Centrilobular emphysema, which we know is frequently associated with cigarette smoking, was also reported in this last CT. We cannot also affirm if the opacities seen were new/active or persistent/chronic, given the fact that they overlapped with those described in the previous CT scan. Regardless, we think that the involvement of the CNS was more exuberant than any other involvement, making it a challenge for us.

In conclusion, diagnosis of ANCA associated CNS vasculitis is challenging and should be suspected in patients with AAV if there is clinical evidence of cerebral involvement and if MRI shows multiple ischemic lesions and vascular branches irregularities. Awareness of this rare but severe complication is critical for timely recognition and prompt treatment.

Disclosure of potential conflicts of interest: none declared.

\section{References}

1. Jennette JC, Falk RJ, Bacon PA, et al. 2012 Revised International Chapel Hill Consensus Conference Nomenclature of Vasculitides. Arthritis Rheum. 2013 Jan;65(1):1-11.

2. Jennette JC, Nachman PH. ANCA Glomerulonephritis and Vasculitis. Clin J Am Soc Nephrol 2017;12(10):1680-1691.

3. Yates M, Watts R. ANCA-associated vasculitis. Clin Med (Lond). 2017;17(1):60-64.

4. Lionaki S, Hogan SL, Jennette CE, et al. The clinical course of ANCA small-vessel vasculitis on chronic dialysis. Kidney International 2009;76:644-651.

5. Kemna MJ, Damoiseaux J, Austen J, Winkens B, Peters J, van Paassen P, Cohen Tervaert JW. ANCA as a predictor of relapse: Useful in patients with renal involvement but not in patients with nonrenal disease. J Am Soc Nephrol. 2015 Mar;26(3):537-542.

6. Jayne DR, Gaskin G, Pusey CD, Lockwood CM. ANCA and predicting relapse in systemic vasculitis. QJM 1995;88(2):127-133.

7. Drachman DA. Neurological complications of Wegener's granulomatosis. Arch Neurol 1963;8(2):145-155.

8. Ghinoi A, et al. Anti-neutrophil cytoplasmic antibody (ANCA)-associated vasculitis involving the central nervous system: case report and review of the literature. Clin Exp Rheumatol 2010;28(5):759-766.

9. Nishino H, Rubino FA, Deremee RA, Swanson JW, Parisi JE. Neurological involvement in Wegener's granulomatosis: an analysis of 324 consecutive patients at the Mayo Clinic. Ann Neurol 1993;33(1):4-9.

10. Seror R, Mahr A, Ramanoelina J, Pagnoux C, Cohen P, Guillevin L. Central nervous system involvement in Wegener granulomatosis. Medicine (Baltimore) 2006;85(1):54-65.

11. Chen M1, Yu F, Zhao MH. Relapses in patients with antineutrophil cytoplasmic autoantibodyassociated vasculitis: likely to begin with the same organ as initial onset. J Rheumatol 2008;35(3):448-450.

12. Murphy JM, Gomez-Anson B, Gillard JH et al. Wegener granulomatosis: MR imaging findings in brain and meninges. Radiology 1999;213(3):794-799.

13. Reinhold-Keller E, de Groot K, Holl-Ulrich K et al. Severe CNS manifestations as the clinical hallmark in generalized Wegener's granulomatosis consistently negative for antineutrophil cytoplasmic antibodies (ANCA). A report of 3 cases and a review of the literature. Clin Exp Rheumatol 2001;19(5):541-549.

14. Sonneville $R$, Lagrange $M$, Guidoux $C$, et al. The association of cardiac involvement and ischemic stroke in Churg Strauss syndrome. Rev Neurol (Paris) 2006;162(2):229-232.

15. Marra AM, Barilaro G, Villella V, Granata M. Eosinophilic granulomatosis with polyangiitis (EGPA) and PRES: a case-based review of literature in ANCA-associated vasculitides. Rheumatol Int 2015;35(9):1591-1595.

16. Patel UV, Patel NJ. Posterior reversible leukoencephalopathy syndrome as a presenting manifestation of p-ANCA-associated vasculitis. BMJ Case Rep 2014; 22, 2014.

17. Fuentes AG, Komarla A, Gomez Jl. Posterior reversible encephalopathy syndrome in a patient with ANCA-associated vasculitis. Rheumatol Int 2012;32(8):2529-2530.

18. Tajima Y, Matsumoto A. Reversible posterior leukoencephalopathy syndrome in p-ANCA-associated vasculitis. Intern Med 2006;45(20):1169-1171. 
19. Salvarani C, Brown RD JR, Calamia KT, et al. Primary central nervous system vasculitis: analysis of 101 patients. Ann Neurol 2007;62(5):442-451.

20. Larry B. Goldstein, MD. Primary Central Nervous System Vasculitis: Is Biopsy Appropriate? Ann Neurol 2008;64(2):228.

21. Pettersson E, Heigl Z: Antineutrophil cytoplasmic antibody (cANCA and pANCA) titers in relation to disease activity in patients with necrotizing vasculitis: a longitudinal study. Clinical Nephrol 1992;37(5):219-228.

22. Tomasson G, Grayson PC, Mahr AD, Lavalley M, Merkel PA: Value of ANCA measurements during remission to predict a relapse of ANCA-associated vasculites - A meta-analysis. Rheumatology (Oxford) 2012;51(1):100-109.

23. Tervaert JWC, Damoiseaux J: Antineutrophil cytoplasmic autoantibodies: How are they detected and what is their use for diagnosis, classification and follow-up? Clin Rev Allergy Immunol 2012;43(3):211-219.

24. Pinching AJ, Rees AJ, Pussell BA, Lockwood CM, Mitchison RS, Peters DK. Relapses in Wegener's granulomatosis: the role of infection. Br Med J 1980;281(6244):836-838.
25. Stegeman CA, Tervaert JW, de Jong PE, Kallenberg CG. Trimethoprim-sulfamethoxazole (co-trimoxazole) for the prevention of relapses of Wegener's granulomatosis. Dutch Co-Trimoxazole Wegener Study Group. N Engl J Med 1996;335(1):16-20.

\section{Correspondence to:}

Sara Rodrigues, MD

Centro Hospitalar Vila Nova de Gaia e Espinho - Unidade 1,

Serviço de Nefrologia - Pavilhão Satélite,

Rua Conceição Fernandes 1079,

Postal Code: 4434-502, Vila Nova de Gaia, Portugal,

E-mail: danisrodrigs@gmail.com. 